\title{
Design on Item Bank System of Vocabulary Comprehension for English Network Examination
}

\author{
XueFeng Liu
}

Teaching and Research Institute of Foreign Languages, Bohai University, Jinzhou, 121013, China

haoxiangchun2005@163.com

Keywords: English; network examination; vocabulary comprehension; item bank; relationship class diagram; functional structure; selected examination question

\begin{abstract}
The effective ways to improve the correct rate of our English vocabulary comprehension is conducting a large number of simulation exam through networks, the content of this research has laid the foundation of for the development of network simulation exam system. First of all, relationship class diagram design, designs a class diagram composed of three entities such as "Examination question", "Alternative vocabulary" and "Standard answer"; Then, functional structure design, expressed the system module composition by the method of function structure diagram; Finally, designed selected examination question process, expressed it through the flow chart randomly, and explained the selected process with the usage of natural language. According to vocabulary comprehension database system constructed in the research contents of this paper can provide abundant information of questions, and finally achieve the purpose of improving the students' comprehensive application level and examination performance.
\end{abstract}

\section{Introduction}

University English teaching is an organic part of higher education, University English course is a compulsory and basic course for college students, and the teaching goal is to train students' comprehensive application ability [1]. vocabulary comprehension mainly examines the students' comprehension to the overall characteristics of continuity, consistency, logical etc. and the words in the context, requires examinees to grasp the macro structure of the article and microscopic comprehension about each word on the basis of the comprehension of the full text, it is not simply examining words' sound, shape, righteousness, but pay more attention to ability of practical application of vocabulary [2]. The effective method to improve the vocabulary comprehension correct rate is conducting mock exam often. Through a large number of network simulation test, to improve the students' competence of lexical application and discourse comprehension, find out the rules about solving problems, grasp the answering steps, pay more attention to grammar learning, have the awareness of discourse, make the reasonable arrangement of time in accordance with the answer, make a adjustment of vocabulary learning method timely, make full use of spare time step by step, completed a lot of practice dependably, earnestly and independently, you should fumble summarize, reflect, improve constantly and can be handy to do this kind of problem. In this paper, the study in order to meet the needs of students' network simulation examination, lays the foundation for the development of vocabulary comprehension item bank system for English network examination.

\section{Design on Relationship Class Diagram}

Vocabulary comprehension system consists of three entities, respectively is the "Examination question", "Alternative vocabulary" and "Standard answer". Between "Examination question" and "Alternative vocabulary" is "one to many" association, that is a question with multiple choice of vocabulary, a alternative vocabulary only corresponding to a question; Between "Examination question" and "Standard answer" is also "one to many "association, that is the themes of a question as many as the standard answers, a standard answer can only correspond to a question. 
Relations between entities are through the unified modeling language in the class diagram to describe. As a current and modeling language, the UML play s a role in modeling language standard, the formal description of the function is very rich, and plays a role in accurate communication enough [3]. UML is an OMG standard, is a graphical language for a support model and software system development, can be used for the whole process of software development, to provide the model and visualization support for all stages of software development [4]. Class is the most important tectonic blocks in object oriented systems. The class diagram shows a set of classes, interfaces, coordination and the relationship between them. In the UML, problem domain was transformed eventually and gradually, using the class to model, using the programming language to construct these classes to implement system. Classes and relations between them constitute the class diagram. Relationship between classes is more complex content in class, where the association is a common relationship, is a kind of semantic relationship among model elements, is a kind of very weak relationship between classes [5]. Association can have a direction, can be one-way connection, and also can be the bidirectional association. And you can make association plus the relation name to describe the relational function. Three entities related class diagram of the system as shown in Fig. 1.

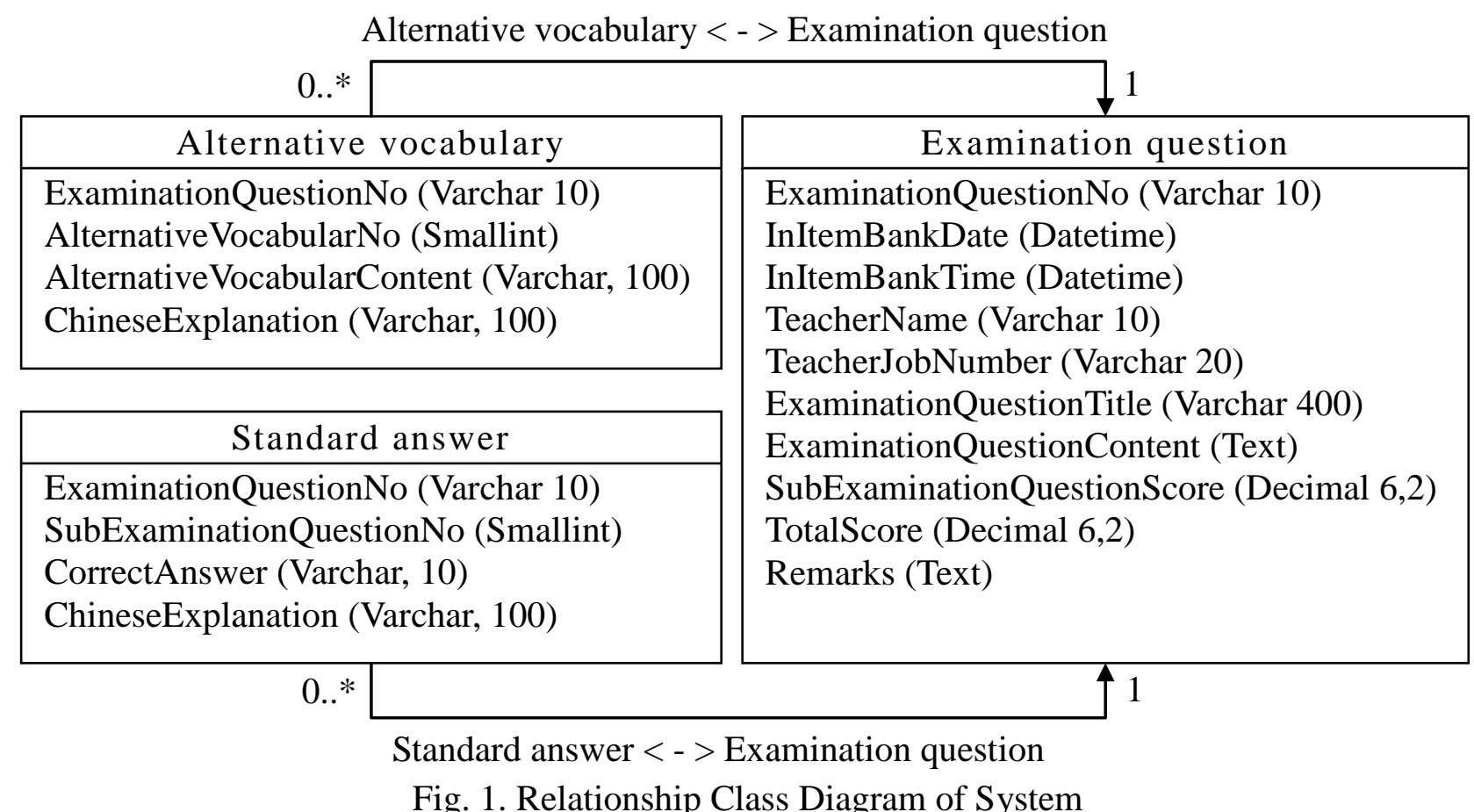

\section{Design on Functional Structure}

The common method of functional structure design is simulation. Modularity is a main thinking for designing, a complex system is decomposed into some smaller scale, simpler function, more easy to set up and modify parts. On the one hand, each module has its own relative independence, can respectively design and implement; on the other hand, to explain the relationships between the modules through a certain way. The common modules constitute a unified whole in these constraints of relationships, complete every function of the system.

The process of functional structure design is the process of function decomposition; the design result is represented by the function structure diagram. The structure chart of the function is expressed by function dependency graphs, and every rectangle in graphs is referred to as a function module. Each subsystem of the management information system can be regarded as the target function of lower layer system, in which each function could continue for more function decomposition. Conceptually, the upper function control the lower, the more upper the more generally, the more lower the more specific. Functional decomposition is a process from abstract to 
concrete, from simple to complex. Design of function and structure of the system is only a level of functional decomposition, as shown in Fig. 2.

\begin{tabular}{|c|c|}
\hline \multicolumn{2}{|c|}{ Vocabulary comprehension item bank system of English network examination } \\
\hline Examination question append & Re create index \\
\hline Examination question update & Common information maintenance \\
\hline Examination question delete & System parameter setting \\
\hline Examination question query & Database backup \& recovery \\
\hline Examination question extraction & \\
\hline Test paper generation & Modify user password \\
\hline Data statistical report & Online help and guide \\
\hline
\end{tabular}

Fig. 2. Functional structure on vocabulary comprehension item bank system of English network examination

In Fig. 2, The left part is the operation of the test question database, including the increase, modify, delete, query, as well as test questions, test paper generation and data statistics report; On the right side is the operation of the system maintenance, Including the re creation index, common information maintenance, system parameter settings, modify user password, data backup and recovery, user and its authority management, online help and use guide, etc..

\section{Design on Selected Examination Question Process}

Selected examination question is selecting the question from the item bank to meet the constraints of the difficulty coefficient, knowledge point and answer time. Because of the existence of a large number of questions in the item bank, uses the certain algorithm to extract to meet the requirements of the test. In this paper, using the method of stochastic algorithm based on random sampling. Random sample is randomly selected questions according to the paper standards which have been identified, eventually combined into paper, which is a common algorithm for the process of selected examination question. Random sampling method is the transfer process which is repeated constantly, until the selected process is completed or can not selected questions to meet users' needs. Random sampling method is very simple process, can not be retrospective and so on, and is currently the most widely algorithm on selected questions. In the use of random functions, using random function provided by the programming language or random number of system time series in usually pumping problem [6, 7]. The process of random selected method is as shown in Fig. 3.

The process of selected examination question is described as follows:

The first step: read parameters from "parameter setting table", set $i=1$, and $i$ is question species variable.

The second step: if $i \leq M$, and there is no test questions, then the next step; Otherwise, turn to the end.

The third step: set $i=i+1$, set $j=1$, $\mathrm{j}$ is the variable number of questions of $i$ type.

The fourth step: if $j \leq N_{i}$, the number of questions of $i$ type does not meet the requirements, then the next step; otherwise, return to the second step.

The fifth step: set $k=0, k$ is a variable of the selected times of a question.

The sixth step: if $k \leq P$, times of selected questions which is not successful are less than $P$, then the next step; otherwise, turn to the end.

The seventh step: generate random numbers, and set $k=k+1$. 
The eighth step: selected questions are not in the "test table", then the next step; otherwise, return to step seventh.

The ninth step: difficulty coefficient of selected question meets the requirements, then the next step; otherwise, return to step seventh.

The tenth step: the knowledge points of selected questions meet the requirements, then the next step; otherwise, return to step seventh.

The eleventh step: the time that the answers taken of selected question meets the requirements, then the next step; otherwise, return to step seventh.

The twelfth step: join the selected question to "test table", if $j=j+1$, then return to the fourth step.

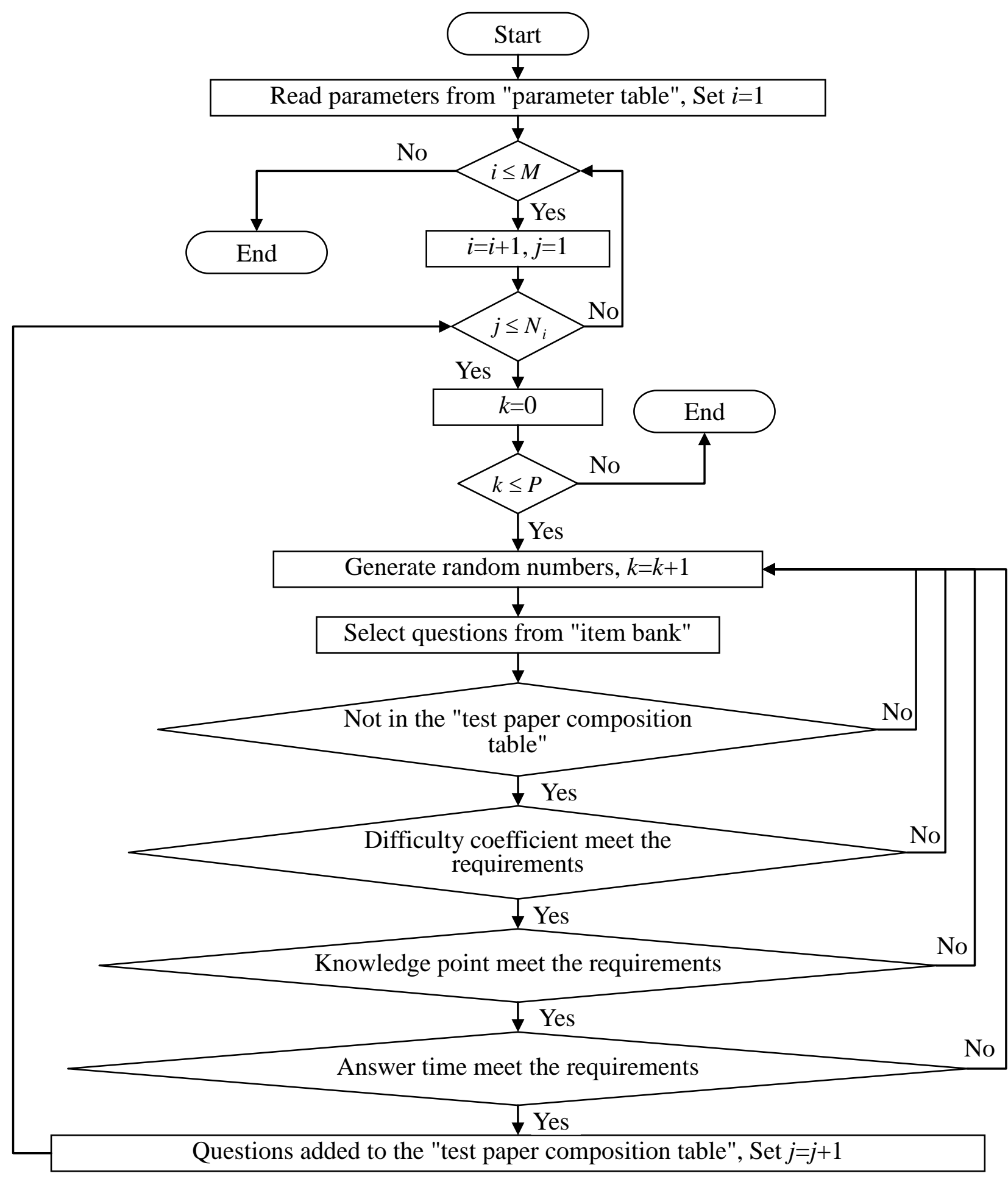

Fig. 3. Random selected examination question process 


\section{Conclusion}

University English teaching should make full use of the network and abundant English teaching resources provides by computer, riches resources for teaching and students' autonomous learning, to create a good English learning environment, make the formation of teaching system which is complete and reasonable [8]. Vocabulary comprehension is a new question type of the English four levels of examination in university, and the goal is to examine students' comprehensive language ability, including the ability of text comprehension, vocabulary application and grammar knowledge $[9,10]$. According to the research contents of this paper build vocabulary comprehension item bank system, which can provide rich information, provide a new measurement method on the basis of the development of network simulation examination, which students can fully understand the general requirements, the way to solve the question, problem-solving methods and skills in this type of question through a number of network simulation examination, and finally achieves the improvement of the students' comprehensive application level and the objective of examination performance.

\section{Acknowledgement}

This work is supported by " Research on personnel training mode for Liaoning province college English second classroom", a general subject of "the Twelfth Five-year Plan" on Educational Science of Liaoning Province, 2012, Approval (JG12DB243).

\section{References}

[1] D. Y. Li, "Implementation strategy on project teaching method in college English teaching reform," Journal of Changchun Education Institute, vol. 30, no. 4, pp. 78-79, 2014.

[2] Y. Yi, "Research on the strategies to improve the university English four level of test questions in the correct rate of text understanding," Theory and Practice of Contemporary Education, vol. 4, no. 12, pp. 149-152, 2012.

[3] J. Z. Liu, H. X. Xia, "New Methods of Software Requirements Risk Assessment Using UML," Computer Science, vol. 41, no. 6, pp. 131-135, 2010.

[4] H. R. Xiao, "Application of software system development based on UML," Journal of Shaanxi University of Technology(Natural Science Edition) vol. 18, no. 4, pp. 274-276, 2006.

[5] Baidu Encyclopedia, "Unified Modeling Language (UML)," http://baike.baidu.com/link?url=OEe2o_FwVPNuQmsjF9bLeMP-rlAw98xjh5cPmwdXM7BnDJb1DRBgYtV4jKgsShQgabU7bW1PPtXJsv1zyadaK, 2015-8-1.

[6] Y. F. Lei, X. F. Lin, "Analysis and Design of Public Mathematics Network Examination System," Journal of Yunnan Normal University (Natural Sciences Edition), vol. 18, no. 4, pp. 274-276, 2006.

[7] Y. Huang, "Design and Implementation of Random Questions Algorithm of Test Database System," Modern Computer, vol. 27, no. 3, pp. 198-200, 2010.

[8]

$$
\text { Huaqiao University, "College }
$$
http://flc.hqu.edu.cn/s/51/t/268/1b/8c/info7052.htm, 2014-9-9.

English

syllabus,"

[9] R. Shi, "Answer skills analysis comprehension vocabulary," Modern Communication, vol. 24, no. 10, pp. 34, 2010.

[10] R. Shi, "Comparison and application of test paper generating algorithm in network examination system," Journal of Huanggang Polytechnic, vol. 17, no. 3, pp. 94-96, 2015. 